\title{
Insight into the X-ray absorption spectra of Cu-porphyrazines from electronic structure theory
}

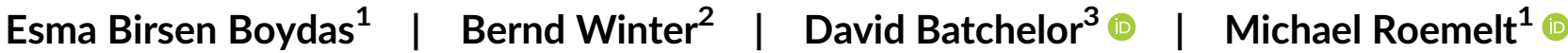

${ }^{1}$ Lehrstuhl für Theoretische Chemie, Ruhr-Universität Bochum, Bochum, Germany

${ }^{2}$ Fritz-Haber-Institut der Max-Planck-

Gesellschaft, Berlin, Germany

${ }^{3}$ Karlsruher Institut für Technologie, Institute for Photon Science and Synchrotron Radiation (IPS), Karlsruhe, Germany

\section{Correspondence}

David Batchelor, Karlsruher Institut für Technologie, Institute for Photon Science and Synchrotron Radiation (IPS), 76021 Karlsruhe, Germany.

Email: david.batchelor@kit.edu

Michael Roemelt, Lehrstuhl für Theoretische Chemie, Ruhr-Universität Bochum, D-44780 Bochum, Germany.

Email: michael.roemelt@theochem.rub.de

Funding information

Deutsche Forschungsgemeinschaft, Grant/

Award Number: RO 5688/1-1

\begin{abstract}
Transition metal porphyrazines are a widely used class of compounds with applications in catalysis, organic solar cells, photodynamic therapy, and nonlinear optics. The most prominent members of that family of compounds are metallophtalocyanines, which have been the subject of numerous spectroscopic and theoretical studies. In this work, the electronic structure and X-ray absorption characteristics of three $\mathrm{Cu}$ porphyrazine derivatives are investigated by means of modern electronic structure theory. More precisely, the experimentally observed $\mathrm{N} \mathrm{K}$-edge and $\mathrm{Cu}$ L-edge features are presented and reproduced by time-dependent density functional theory, restricted open-shell configuration interaction, and a restricted active space approach. Where possible, the calculations are used to interpret the observed spectroscopic features in terms of electronic transitions and, furthermore, to connect spectral differences to chemical variations. Part of the discussion of the computational results concerns the impact of various parameters and approximations that are used for the calculations, for example, the choice of active space.
\end{abstract}

\section{KEYWORDS}

electronic structure theory, transition metal chemistry, $x$-ray absorption spectroscopy

\section{1 | INTRODUCTION}

X-ray absorption spectroscopy (XAS) is a powerful inner-shell spectroscopic technique that is able to probe the electronic structure of molecules and solids alike. During the experiment, an electron from an inner shell is promoted to the valence shell or the continuum. Hence, the energy range for the required X-ray beam is governed by both the specific element and the inner shell that the electron originates from. Consequently, all experimentally observed features are labeled according to these two parameters. For example, the absorption bands that mainly correspond to transitions from the $1 \mathrm{~s}$ shell of a $\mathrm{Cu}$ atom are referred to as $\mathrm{Cu}$ K-edges. Owing to its element specificity, XAS is particularly well suited to investigate the local electronic properties of transition metals even in large ligand environments such as metalloproteins. Depending on the experimental technique used, different properties are accessible, including the metal oxidation state, electronic configuration, spin symmetry, the ligand environment, and metal-ligand bond covalency. However, it has been recently found that an immediate interpretation of absorption data (ie, total absorption yields) might be problematic, in which case it is advisable to rely on various types of partial-yield spectra. ${ }^{[1]}$

Ligand K-edge features correspond to ligand $1 \mathrm{~s}$ to $n p(n=2,3 \ldots)$ electric dipole-allowed transitions, whose intensity mainly maps the ligand p-orbital character in partially occupied and unoccupied molecular orbitals. Consequently, the ligand K-edge intensity is widely acknowledged as a measure of metal-ligand covalency. ${ }^{[2-4]}$ Nowadays, the interpretation and quantitative analysis of ligand K-edge spectra can be efficiently assisted 
by quantum chemical calculations. More precisely, the calculation of ligand K-edge features with time-dependent density functional theory (TDDFT) allows for a relatively simple and straightforward means to assign experimentally observed features to electronic transitions. ${ }^{[4,5]}$

Experimentally, much sharper and richer features are detected for transition metal L-edges that correspond to dipole-allowed $2 p$ to valence transitions. However, the interpretation of these spectra is significantly more difficult as the manifold of target states features not only two open shells-one on the $2 p$ shell and one in the valence shell-but is also subject to strong $2 p$ spin-orbit coupling (SOC) effects due to the $2 p$ core hole. Accordingly, any attempt to model transition metal L-edges in the framework of simple particle-hole models (like TD-DFT) is deemed to fail to reproduce the many occurring multiplets of states and their nontrivial couplings, except for a few simple cases. ${ }^{\left[{ }^{6]}\right.}$ Over the years, several alternative approaches have been developed that explicitly take into account the multiplet structure of the target states' state manifold. ${ }^{\text {[6-12] }}$ One of them is the combination of DFT with restricted open-shell configuration interaction with single excitations (DFT/ROCIS), introduced by Neese et al. It has been shown to yield reasonable results for a variety of different transition metal compounds, even for solids. ${ }^{[6,13-18]}$ Perhaps the most rigorous approach to the problem is based on the restricted active space configuration interaction (RASCI) ansatz. ${ }^{[19-27]}$ Within this approach, the set of molecular orbitals is divided into the internal orbitals that are doubly occupied; the virtual orbitals that are empty; and finally, two sets of active orbitals that are allowed to have a more involved occupation pattern. The first set of active orbitals comprises the transition metal $2 p$ orbitals from which a single electron is promoted into the second set of active orbitals that correspond to a selection of valence orbitals. A predefined number of states with varying multiplicity is then calculated as a linear combination of all possible configuration state functions (or determinants) within the active space with the correct number of electrons and the desired spin symmetry. The required expansion coefficients are obtained from solving a secular equation that results from minimization of the state energies according to the variational principle. Afterward, SOC between the different multiplets is accounted for in a Russell-Saunders-type fashion. It has been found, however, that accurate results are only achieved when dynamic electron correlation is taken into account. ${ }^{[27]}$

In this work, the electronic structure and X-ray absorption characteristics of $\mathrm{Cu}(\mathrm{II})$-porphyrazine scaffolds are investigated. Porphyrazines, or tetraazaporphyrins (usually abbreviated as Pz or TAP), consist of two pyrrole-based groups linked together via a meso-tetraaza substitution. Phthalocyanines (Pcs) are porphyrazine analogs consisting of 18 pi-electrons, with four benzo units fused to the pyrrole moieties (see Scheme 1). Metallophthalocyanines (MPcs) belong to one of the most widely investigated macrocycles due to their chemical value in catalysis, organic solar cells, photodynamic therapy, and nonlinear optics. ${ }^{[29-32]}$ Much less investigated albeit equally prominent derivatives of porphyrazines are napthalocyanines $(\mathrm{Nc})$ with a benzolation degree of 2 . The studied set of molecules presents a rare opportunity to study the spectroscopic effects of a controlled and systematic variation of the ligand $\pi$-system in a transition metal compound. In the following, the experimentally observed $\mathrm{N} \mathrm{K}$ edge and $\mathrm{Cu}$ L-edge features of the three Cu porphyrazine derivatives shown in Scheme 1 will be presented and then simulated by quantum chemical calculations. Where possible, the calculations are used to interpret the observed spectroscopic features in terms of electronic transitions and, furthermore, to connect spectral differences to chemical variations and their concomitant changes on the local electronic structure at the $\mathrm{Cu}$ and $\mathrm{N}$ atoms. Part of the discussion of the computational results concerns the impact of various parameters and approximations that are used for the calculations, for example, the choice of active space.

\section{2 | EXPERIMENTAL DETAILS}

NEXAFS measurements of the Cu porphyrazines depicted in Scheme 1 were made at BESSYII. ${ }^{[28,33,34]}$ The samples were prepared by in situ sublimation on to thin polycrystalline gold films supported on mica substrates. The preparation and initial measurements were carried out in a UHV vacuum chamber on the dipole magnet plane grating monochromator (PGM) beamline PM3. Nitrogen K-edge NEXAFS was measured using Auger electron excitation. The thickness was estimated as circa $80 \mathrm{~nm}$ based on calibration using attenuation of Au $4 \mathrm{f}$ photoemission. The spot size was

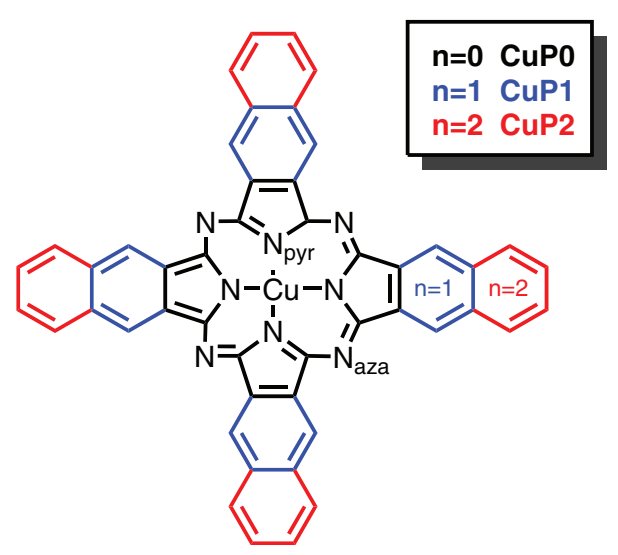

SCHEME 1 Copper porphyrazine derivatives CuPO to CuP2 investigated in this work. The nomenclature follows previous works on structurally similar compounds ${ }^{[28]}$ 
of the order of $1 \mathrm{~mm}$ to avoid beam damage and so was also averaged over any grains present (spectra from different spots showed negligible variation). No charging was observed in the porphyrazine valence or core-level photoemission supporting a relatively thin deposited film. A short angular investigation showed no changes in the NEXAFS structure with X-ray incidence angle, indicating an isotropic excitation of the molecules as would be obtained by an average over many randomly orientated crystals or in amorphous films: A preferred orientation would typically manifest itself in the anticorrelated behavior of intensities in the $\sigma$ and $\pi$ resonances. ${ }^{[3,36]}$ The absence of a preferred orientation is attributed to a rapid growth rate, polycrystalline substrate, and the tert-butyl alkyl substituents to the benzene group, hindering $\pi$-stacking interaction of the molecules. Comparison of NEXAFS repeat scans at different spots and times showed homogeneously grown films and the absence of beam damage. Similarly, the photoemission indicated no damage. The samples were transferred to the Cottbusser BTUC Undulator PGM beamline, ${ }^{[37]}$ where they were subsequently measured using a low-energy-resolution fluorescence detector (EDX) to compare surface and bulk information. Here, the nitrogen edge measurements evidenced a small pre-edge dip due to self-absorption, which could simply be corrected for. ${ }^{\left[{ }^{38]}\right.}$ Both raw and corrected Nitrogen NEXAFS showed the same structure as the data taken at the dipole beamline PM3. The higher photon energy Copper $\mathrm{L}_{2,3}$ NEXAFS showed no self-absorption problem due to the much higher attenuation length of these photons. Small beam damage was observed after several scans for the much higher flux density undulator beamline. In all shown measurements, the resolution of the spectra was limited by the sample and not beamline (Undulator PGM resolving power of $\frac{E}{\Delta E} \rightarrow 10000$ ).

\section{3 | COMPUTATIONAL METHODOLOGY}

In this work, multiple sets of calculations on different levels of theory were conducted, the majority of which used the ORCA program package version 4.2. ${ }^{[39]}$ Geometry optimizations were performed utilizing the global hybrid B3LYP density functional along with def2-TZVP(-f) basis set. ${ }^{[40-42]}$ Accuracy in numerical integration was ensured by a dense grid (ORCA Grid5). The generation of Coulomb and exchange integrals was accelerated with the resolution of identity (RI) and chain-of-sphere (COSX) approximations, ${ }^{[43]}$ together with the def2/J basis set. ${ }^{[44-47]}$

Nitrogen K-edge XAS pre-edges were modeled by TD-DFT ${ }^{[48]}$ calculations, where electrons were only allowed to be excited from molecular orbitals with a predominant (>99\%) N 1s character. As the core-hole created by the X-ray radiation is localized on a single atom, the $\mathrm{N} 1 \mathrm{~s}$ orbitals were localized according to the Pipek-Mezey scheme to truly mimic the underlying phenomena. ${ }^{[49]}$ During all TD-DFT calculations, the TammDancoff approximation was used as implemented in the ORCA program package. ${ }^{[5,50]}$ Scalar relativistic effects were approximated by means of the zeroth-order regular approximation. ${ }^{[51,52]}$ Calculated spectra were obtained by convoluting the calculated transitions with Gaussian functions with a width of $2.0 \mathrm{eV}$. Finally, all calculated spectra are shifted by $\sim 12 \mathrm{eV}$ to allow for a better comparison with the experimental analogs. This shift is necessary to account for the systematic errors introduced by the TD-DFT approach and depends on the chosen functional and basis set. ${ }^{\text {[5] }}$

The presented calculations of $\mathrm{Cu}$ L-edge spectra by means of the above-mentioned restricted active space approach included a sequence of different steps. First, the valence active space was selected as described below with the help of the recently introduced active space selection based on 1st-order perturbation theory (ASS1ST) scheme that is implemented in the MOLBLOCK program. ${ }^{[54,55]}$ Then, all molecular orbitals were optimized with respect to a large number of valence excited states (up to $12 \mathrm{eV}$ ), as suggested previously. ${ }^{[25]}$ In the following, the conventional ( $m, n$ ) notation for active spaces will be adopted, where $m$ is the number of active electrons, and $n$ is the number of active orbitals. After the orbital optimization procedure, the $\mathrm{Cu} 2 \mathrm{p}$ orbitals were appended to the active space to provide a final active space that allows for the evaluation of core excited-state calculations with a $\left(2 p^{5}\right)$ (valence ${ }^{N+1}$ ) configuration at the $\operatorname{RASCI}(m, n)$ level of theory. Importantly, the $K$-calculated RASCI states were divided into ${ }^{2} \mathrm{~K}$ states with a total spin of $S=\frac{1}{2}$ and ${ }^{4} K$ states with $S=\frac{3}{2}$. The latter set needs to be taken into account because the $2 p$-to-valence excitation potentially leads to two additional unpaired electrons compared to the electronic ground state. All $K={ }^{2} K+{ }^{4} K$ state energies were then refined by taking into account dynamic electron correlation effects with second-order n-electron valence perturbation theory (NEVPT2) in its strongly contracted variant. ${ }^{[56,57]}$ Finally, SOC effects were modeled in the framework of quasidegenerate perturbation theory, which can be regarded as a molecular equivalent to atomic Russell-Saunders coupling. ${ }^{[6]}$ The widely used spin-orbit mean-field approximation was employed during the evaluation of SOC matrix elements. ${ }^{[58]}$ All steps involved in the Cu L-edge spectra calculations used the second-order Douglas-Kroll Hess (DKH2) correction to include scalar relativistic effects. ${ }^{[42,59,60]}$ Accordingly, the cc-pVTZ-DK basis set was chosen. ${ }^{[61-63]}$ Two-electron integrals were evaluated using the RI approximation together with an automatically generated auxiliary basis set. ${ }^{[64]}$ At this point, it should be noted that all presented calculations correspond to the gas phase and therefore neglect solvent effects or interactions with neighboring sample molecules altogether.

\section{4 | RESULTS AND DISCUSSION}

\section{1 | Electronic ground state of CuPO-CuP2}

The porphyrazine structures investigated herein adopt a square-planar geometry with a $\mathrm{D}_{4 \mathrm{~h}}$ point group symmetry. As shown in Scheme 1 , the central $\mathrm{Cu}$ ions are coordinated by four pyrrolic nitrogen atoms. The four pyrrole units are connected to a single tetradentate ligand by four 


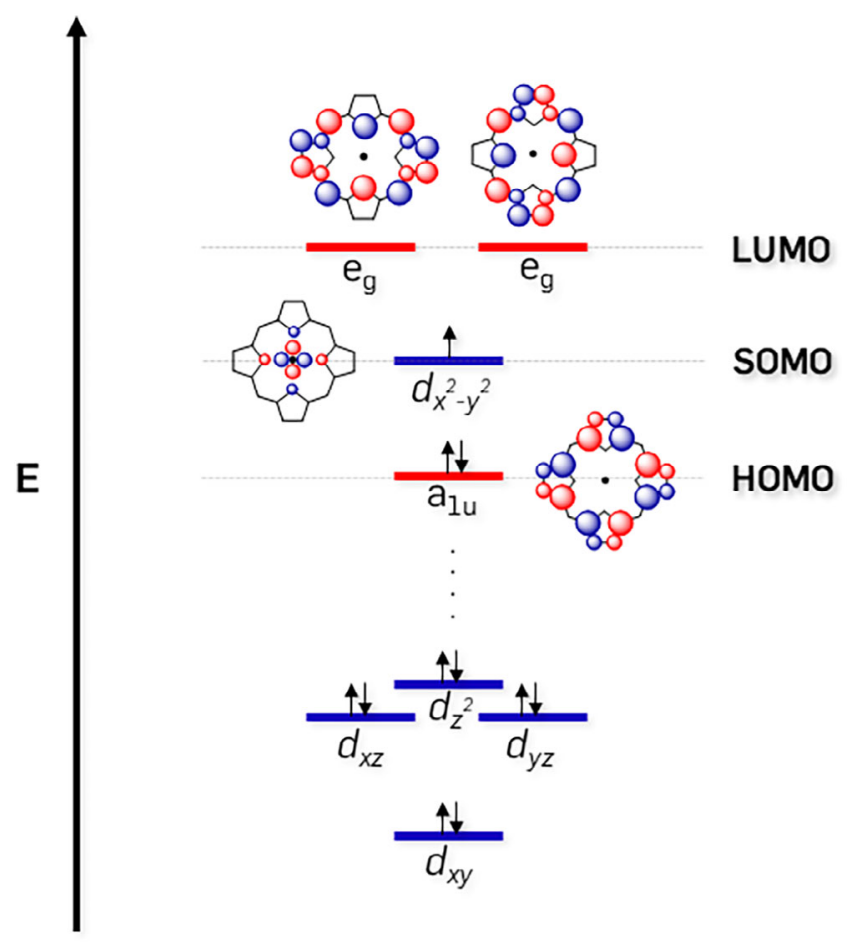

FIGURE 1 Schematic representation of orbital energy levels for $\mathrm{Cu}$ porphyrazine derivatives taking into account the $\mathrm{Cu} 3 \mathrm{~d}$ shell and the ligand-centered frontier orbitals. Blue bars are used for orbitals with a predominant metal $d$ character, while red bars indicate mostly ligandcentered orbitals

UMO

SOMO

additional nitrogen atoms in meso position. Consequently, two nonequivalent sets of nitrogen atoms labeled $\mathrm{N}_{\text {pyr }}$ and $\mathrm{N}_{\text {aza }}$ can be distinguished (see Figure 1). The nomenclature of nonequivalent nitrogens has been adapted from a previous theoretical study on metal Pcs. ${ }^{[65]}$ Further information on critical bond distances of $\mathrm{Cu}-\mathrm{N}_{\text {pyr }}$ and $\mathrm{N}_{\mathrm{aza}}-\mathrm{C}$ is included in Supporting Information.

The tetragonal ligand field imposed by the porphyrazine ligand causes a splitting of the Cu d-orbital energies as shown in Figure 1 . In line with previous works on the structurally related Pcs, we find that the molecular orbital corresponding to the Cu $3 d_{x^{2}-y^{2}}$ orbital is considerably higher in energy compared to the rest of the $\mathrm{Cu} 3 \mathrm{~d}$ orbitals on account of the strong interactions with all four coordinating N atoms. ${ }^{[66]}$ In agreement with the oxidation state of II on the central Cu atom, this orbital is only singly occupied in the leading configuration of our CASSCF (7,7) calculations (see below) of the ground state of CuPO-CuP2. Hence, the electronic ground state for all cases is dominated by a Cu (3d ${ }^{9}$ ) configuration and therefore exhibits ${ }^{2} B_{1 g}$ symmetry. As expected, the first two unoccupied orbitals are a pair of degenerate $\pi^{*}$ orbitals that resemble the unoccupied part of the so-called Gouterman orbitals of metalloporphyrins. ${ }^{[67]}$ In contrast to the Gouterman 4-orbital model, however, the pair of ligandcentered $\pi$ orbitals that correspond to highest occupied molecular orbital (HOMO) and HOMO-1 in the present cases lose their degeneracy when the macrocycle is endowed with a central metal ion.

\section{2 | Nitrogen K-edge XAS}

A comparison of experimental and calculated XAS spectra of $\mathrm{N} 1 \mathrm{~s}$ core excitations for all three investigated molecules is given in Figure 2. The oscillator strengths of each calculated transition are represented by sticks, which provide additional insight into the peak structures. In general, good agreement between the calculated and experimental spectra is observed in the sense that all significant features in the $\mathrm{N} 1 \mathrm{~s}$-to- $\pi^{*}$ region are reproduced well in terms of position and intensity. In all three cases, the most intense peak at about $399 \mathrm{eV}$, labeled as A, gains its intensity from four main transitions. Going from CuPO to CuP2, the energy splitting between these transitions increases. Interestingly, with an increasing degree of benzolation, the number of distinct features beyond this main feature decreases, leading to rather broad bands at energies $\geq 400 \mathrm{eV}$ for CuP2. For example, feature $\mathrm{C}$ at $\sim 402 \mathrm{eV}$ in the spectrum of CuPO appears as a single, intense band, while the spectrum of CuP2 exhibits no such isolated feature.

To reveal the origins of the $\mathrm{N}$ 1s to core excitations, the deconvoluted spectra with respect to the nonequivalent nitrogens are given in Figure 3. Here, the blue and yellow curves correspond to the inner $\mathrm{N}_{\text {pyr }}$ and outer $\mathrm{N}_{\text {aza }}$ nitrogens, respectively. In all cases, the lowest-energy main peak $A$ has contributions from both nonequivalent nitrogens. The lowest-energy transitions for CuPO and CuP1 correspond primarily to the outer nitrogens $\mathrm{N}_{\text {aza }}$. With increasing $\pi$ conjugation, transitions originating from the inner nitrogens $\mathrm{N}_{\text {pyr }}$ progressively shift to lower energies and higher relative intensities. 
FIGURE 2 A comparison of experimental and calculated spectra for $\mathrm{N}$ K-edges. The two sets of spectra agree well up to an excitation energy of $\sim 403 \mathrm{eV}$. The contribution from each transition to the calculated spectra is indicated by a black vertical line
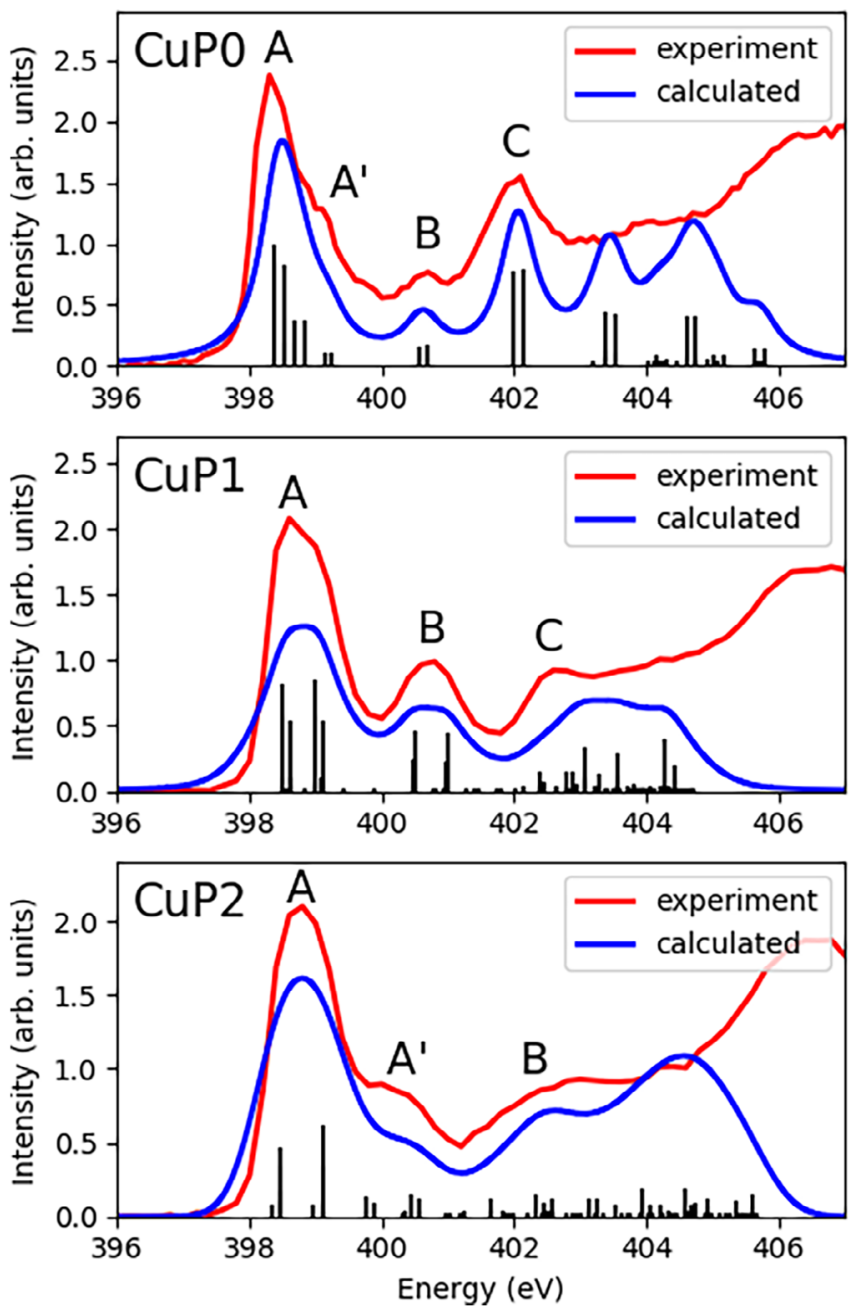

As illustrated by their natural difference orbitals (NDOs) in Figure 4A,B, the transitions corresponding to feature $\mathrm{A}$ in the spectrum of $\mathrm{CuPO}$ have $\mathrm{N} 1 \mathrm{~s}$ to $\pi^{*}$ character. In contrast, the shoulder labeled $\mathrm{A}^{\prime}$ is due to $\mathrm{N} 1 \mathrm{~s}$ to $\pi^{*}$, as well as $\mathrm{N} 1 \mathrm{~s}$ to $\sigma^{*}$, transitions (see Figure $4 \mathrm{C}$-F). Finally, features $B$ and $C$ are dominated by $N$ 1s to $\pi^{*}$ transitions again (see Figure 4I,J). However, in these cases, the acceptor orbitals are located more toward the periphery than for feature A. A similar trend is observed for CuP1 and CuP2 as well. While absorption bands A correspond to transitions into $\sigma^{*}$ and $\pi^{*}$ orbitals that are located in the vicinity of the central $\mathrm{Cu}$ ion, the acceptor orbitals associated with absorption bands B and $\mathrm{C}$ are considerably shifted toward the periphery of the molecules (see Figures 5 and 6). It should be noted that the spectrum of CuP2 at the bottom of Figure 2 is significantly broadened compared to the spectra of CuPO and CuP1 and consequently lacks clear features. In light of our computational results, this finding can be attributed to the increased number of $\pi^{*}$ acceptor orbitals that causes a broader distribution of transitions with similar excitation energy.

\section{3 | Cu L-edge XAS}

\subsection{1 | Experimental spectra}

The experimental L-edge spectra of CuPO and CuP1 are shown in Figure 7, while the corresponding fitted peak positions, intensities, and widths are tabulated in Table 1. We restrict the following discussion to CuPO and CuP1 because the spectra of CuP1 and CuP2 have no visible differences (see Supporting Information), which indicates that both compounds feature similar local structural and electronic properties.

As all dipole-allowed $\mathrm{Cu} 2 \mathrm{p}$ to $\mathrm{Cu} 3 d$ transitions have a $\left(2 p^{5}\right)\left(3 d^{10}\right)$ final state configuration, the $L_{3}$ - and $L_{2}$-edges exhibit only little structure. In particular, the $L_{3}$ - and $L_{2}$-edges of CuP1 feature only a single, sharp band. The same holds true for the $L_{2}$ edge of CuPO. Only the $L_{3}$-edge of CuPO appears to be have a shoulder on its high-energy side, denoted as feature B at $\sim 933.5 \mathrm{eV}$. Owing to its position at $1.5 \mathrm{eV}$ higher than the main edge, it may be due to $2 \mathrm{p}$-3d transitions or be dominated by one or multiple additional metal-to-ligand charge-transfer (MLCT) transitions. 

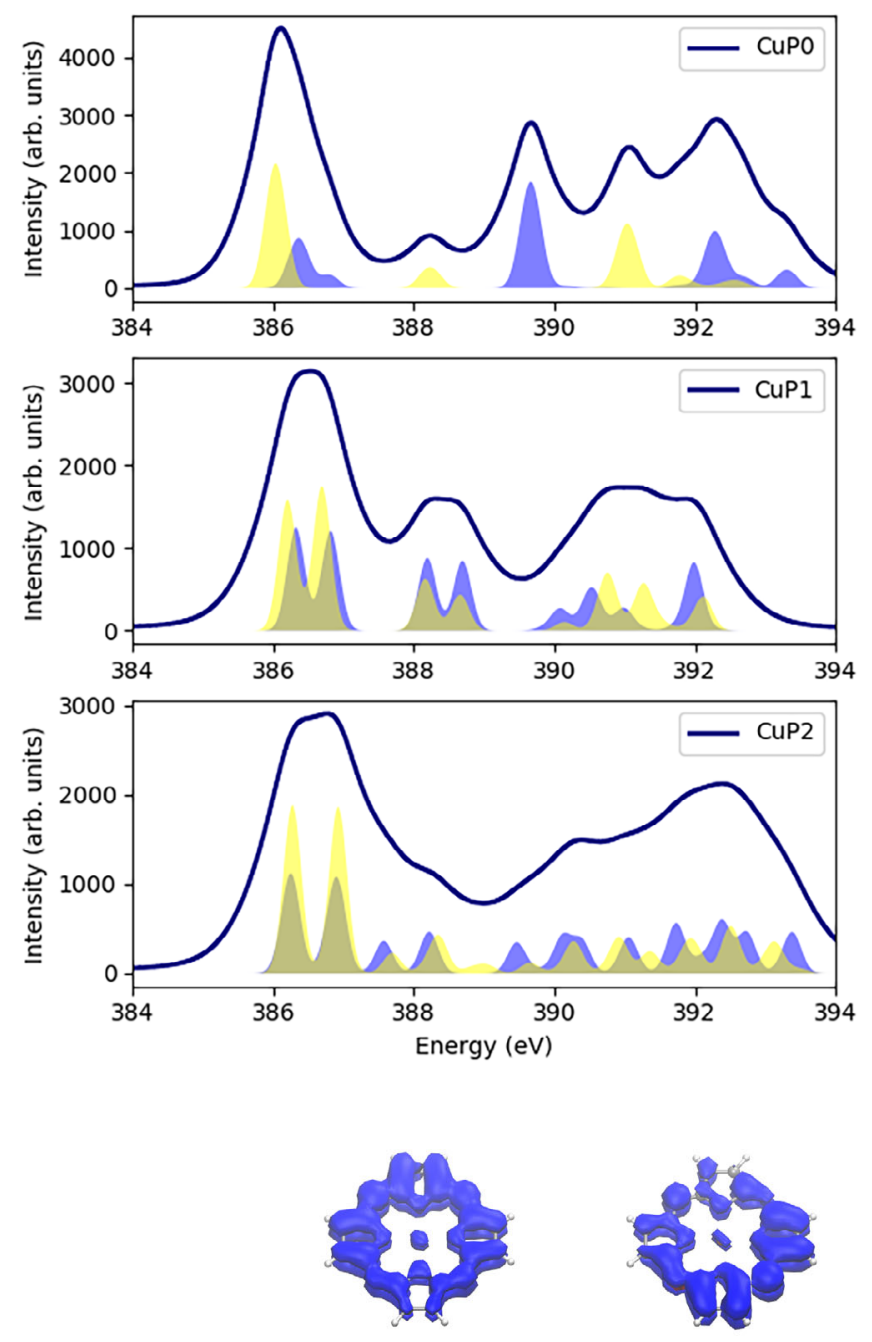

(A) $\mathrm{A}$

(B) $\mathrm{A}$
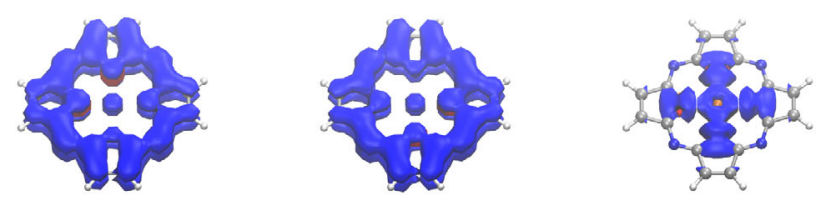

(C) $\mathrm{A}^{\prime}$
(D) $A^{\prime}$

(E) $A^{\prime}$
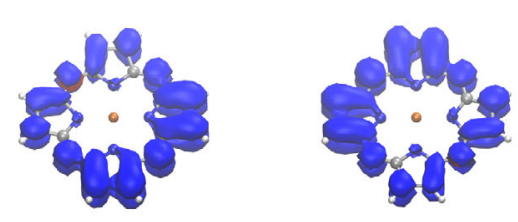

(G) $\mathrm{B}$
FIGURE 3 Deconvolution of total K-edge spectra with respect to $N_{\text {aza }}$ (yellow) and $N_{\text {pyr }}$ (blue)
FIGURE 4 Natural difference orbitals (NDOs) for the relevant transitions for CuPO as labeled in Figure 2

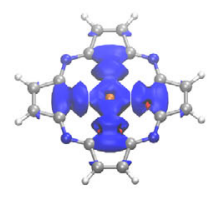

(F) $\mathrm{A}^{\prime}$

A phenomenological approach to elucidate the origin of this feature is to analyze the $L_{3} / L_{2}$ intensity ratio. Depending on whether the shoulder is assigned to the main $\mathrm{L}_{3}$-edge or not, the intensity ratio becomes 2.41 or 1.85 , respectively. A comparison with the intensity ratios of CuP1 and CuP2 (2.31 and 2.17, respectively) suggests that the shoulder is part of the main $L_{3}$-edge and thus mostly originates from $2 p$ to $3 d$ transitions. 

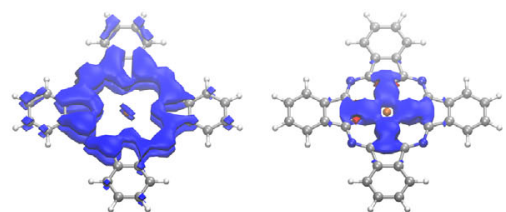

(A) $\mathrm{A}$

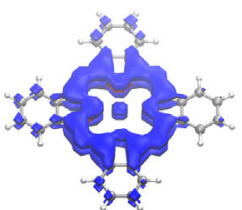

(C) $\mathrm{A}$

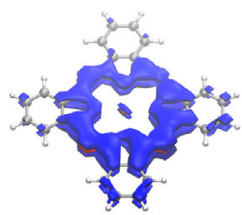

(D) $\mathrm{A}$

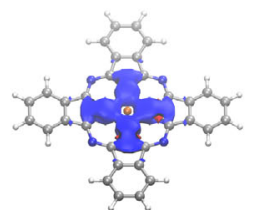

(E) $\mathrm{A}$

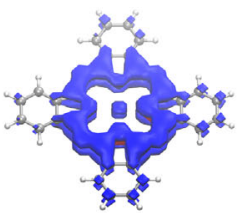

(F) $\mathrm{A}$

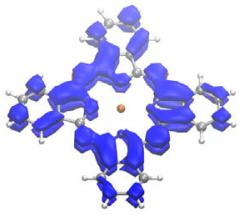

(G) B

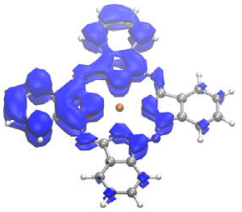

(H) B

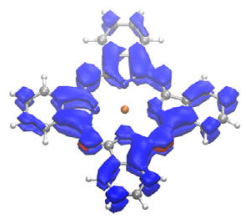

(I) $\mathrm{B}$

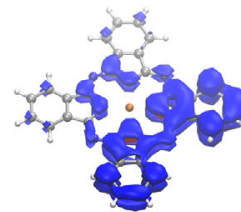

(J) $\mathrm{B}$

FIGURE 5 Natural difference orbitals (NDOs) for the relevant transitions for CuP1 as labeled in Figure 2

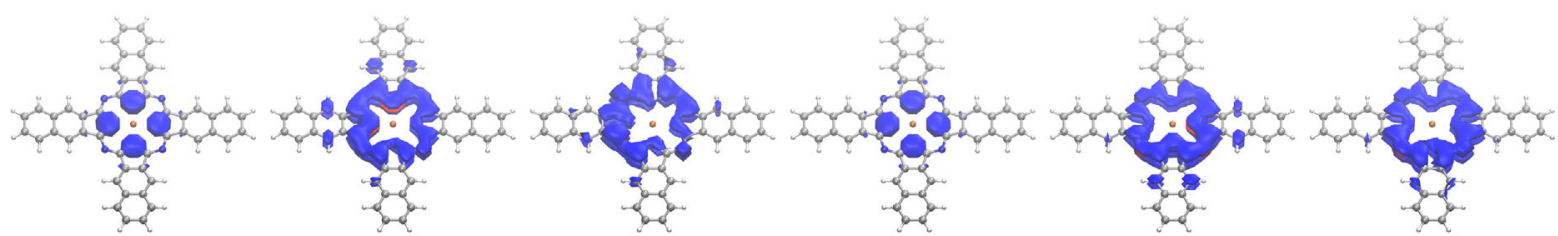
(A) $\mathrm{A}$
(B) $\mathrm{A}$
(C) $\mathrm{A}$
(D) $\mathrm{A}$
(E) $\mathrm{A}$
(F) $\mathrm{A}$
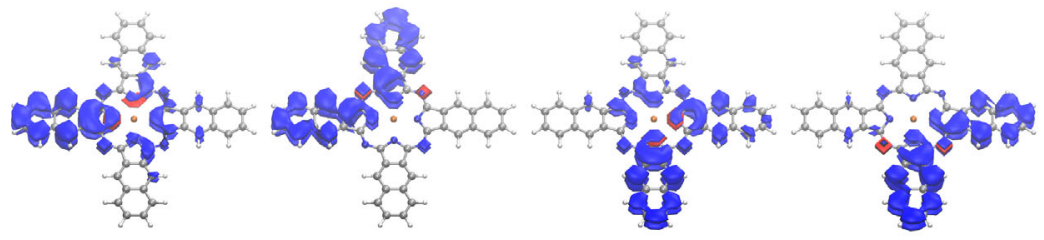

(G) $A^{\prime}$

(H) $\mathrm{A}^{\text {? }}$

(I) $\mathrm{A}^{\text {' }}$

(J) A'
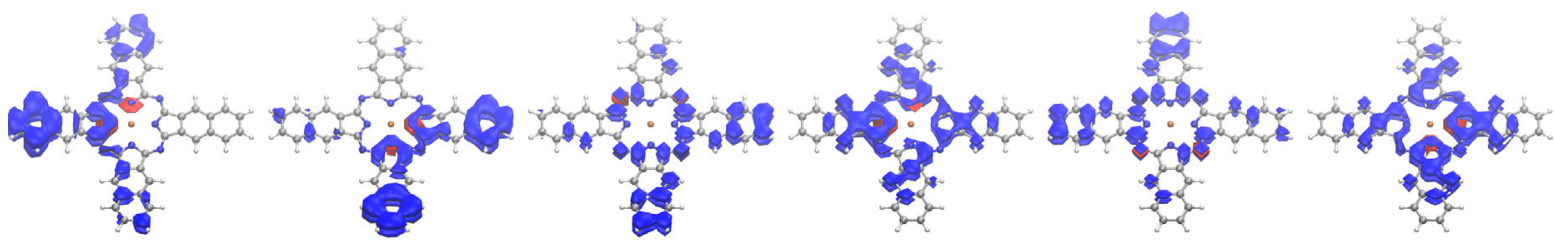

(K) $\mathrm{B}$

(L) $\mathrm{B}$

(M) $\mathrm{B}$

(N) $\mathrm{B}$

(O) $\mathrm{B}$

(P) B

FIGURE 6 Natural difference orbitals (NDOs) for the relevant transitions for CuP2 as labeled in Figure 2

However, the application of a different peak fitting method (see Table 2 and Supporting Information) leads to a less clear picture in this regard. With the alternative fitting method, the $\mathrm{L}_{3} / \mathrm{L}_{2}$ intensity ratio amounts to 2.84 and 2.13 with or without the shoulder, respectively, while CuP1 and CuP2 exhibit values of 2.65 and 2.69 , respectively. Hence, a classification of feature $B$ as originating from a $2 p$ to $3 \mathrm{~d}$ or an MLCT transition solely based on the experimental observations is difficult if not impossible. A further noteworthy difference between the spectra of CuPO and CuP1 is that the main $\mathrm{L}_{3}$-band of CuPO is observed at a $0.43 \mathrm{eV}$ lower energy than its counterpart of CuP1 (932.09 vs $932.52 \mathrm{eV}$ ). Additional weak and broad features are observed at $936.93 \mathrm{eV}$ for $\mathrm{CuPO}$ and at 935.69 and $939.89 \mathrm{eV}$ for CuP1. All features beyond the $\mathrm{L}_{2}$-edge will be omitted from the discussion as this part of the experimental spectrum might be tainted from alternative decay pathways that cannot be properly treated within the current theoretical framework. In the following, the results of quantum chemical calculations on the DFT/ROCIS and RASCI + NEVPT2 levels of theory will be presented with the aim of identifying the origin and character of the observed spectral features of CuPO and CuP1. 


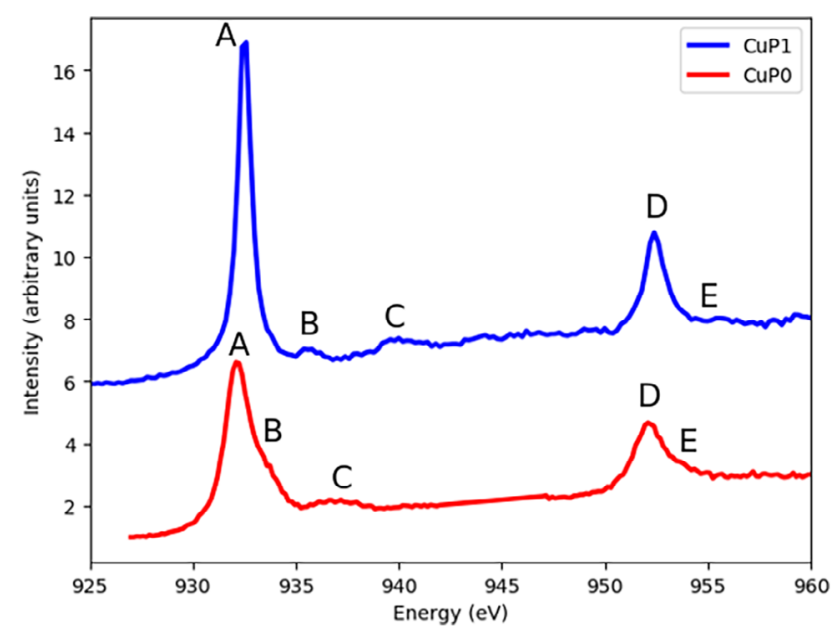

FIGURE 7 Experimentally observed Cu L-edge spectra of CuPO (bottom, red) and CuP1 (top, blue)

\begin{tabular}{lllllllll} 
& \multicolumn{7}{l}{ CuPO } & \multicolumn{2}{l}{ CuP1 } \\
\cline { 2 - 3 } Feature & Position & Area & Width & & Position & Area & Width \\
\hline A & 932.09 & 10.38 & 1.34 & & 932.52 & 13.55 & 0.81 \\
\hline B & 933.20 & 2.47 & 2.01 & & 935.69 & 0.50 & 1.75 \\
\hline C & 936.93 & 0.87 & 2.01 & & 939.89 & 1.03 & 1.75 \\
\hline E & 952.11 & 5.40 & 1.61 & & 952.43 & 5.90 & 1.09 \\
\hline
\end{tabular}

TABLE 1 Fitted peak positions, intensities, and widths for the experimentally observed Cu L-edges of CuPO and CuP1 using the symmetric Voigt lineshape

Note: All energies are given in $\mathrm{eV}$.

\begin{tabular}{|c|c|c|c|c|c|c|}
\hline \multirow[b]{2}{*}{ Feature } & \multicolumn{3}{|l|}{ CuPO } & \multicolumn{3}{|l|}{ CuP1 } \\
\hline & Position & Area & Width & Position & Area & Width \\
\hline A & 932.09 & 1.09 & 1.42 & 932.50 & 1.47 & 0.91 \\
\hline B & 933.20 & 0.33 & 1.87 & 935.51 & 0.09 & 1.53 \\
\hline C & 936.93 & 0.10 & 1.85 & 939.85 & 0.88 & 7.21 \\
\hline D & 952.11 & 0.59 & 1.97 & 952.44 & 0.64 & 1.28 \\
\hline E & 953.82 & 0.19 & 2.89 & 955.44 & 0.08 & 1.63 \\
\hline
\end{tabular}

TABLE 2 Fitted peak positions, intensities, and widths for the experimentally observed Cu L-edges of CuPO and CuP1 using an asymmetric lineshape of a Gaussian convoluted Sunjic-Doniach peak

Note: All energies are given in eV.

\section{\begin{tabular}{l|l} 
4.3.2 & DFT/ROCIS
\end{tabular}}

Figure 8 shows a comparison of the Cu L-edge spectra for CuPO and CuP1 calculated with the DFT/ROCIS method. In agreement with the original work by Neese et al., the calculated transition energies have to be shifted $20.0 \mathrm{eV}$ to match the experimental values. ${ }^{[6]}$ Yet the main objective of this work is to elucidate the physical origin of observed transitions, as well as differential transition energies and band shapes, rather than to reproduce total absorption energies.

Both calculated spectra are remarkably similar as they both feature single intense $L_{3}$ and $L_{2}$ bands originating from $2 p$ to $3 d$ transitions with no visible shoulders. In addition to the main absorption bands, satellite features are predicted at $937.7 \mathrm{eV}$ and $938.0 \mathrm{eV}$ for $\mathrm{CuPO}$ and $\mathrm{CuP} 1$, respectively (see Table 3). As illustrated by the orbital contour plots in the bottom panel of Figure 8 , these features are dominated by transitions into two ligand $\pi^{*}$ orbitals and an MO with mostly $\mathrm{Cu} 4 \mathrm{~s}$ character. The second satellite feature for CuP1 at high energies is absent in the calculated spectrum, and as opposed to the experimental observations, the calculated $\mathrm{L}_{3} / \mathrm{L}_{2}$ intensity ratio is similar for CuPO and CuP1 with values of 1.93 and 1.92, respectively. Furthermore, no shoulder at the high-energy side of the $\mathrm{L}_{3}$-edge of CuPO is visible in Figure 8 . These results, together with the absence of any low-lying MLCT states in this region, indicate that the experimentally observed shoulder on the CuPO $L_{3}$-edge arises from a splitting of the $2 p$ to $3 \mathrm{~d}$ manifold of states rather than an MLCT transition. Nevertheless, these results have to be considered with caution as the splitting of the states could not be reproduced, and the position of the calculated satellite bands is overestimated by 0.7 and $2.4 \mathrm{eV}$ for $\mathrm{CuPO}$ 
FIGURE 8 Experimental and calculated Cu L-edge XAS for CuPO and CuP1 using density functional theory/restricted open shell configuration interaction with single excitations (DFT/ROCIS). The bottom images show enlarged views of the calculated $L_{3}$-edge and the satellite features. Both spectra are remarkably similar with only slight energy shift of the main $\mathrm{L}_{3}$-edge and the satellite peak. While the main absorption feature is due to $2 p \rightarrow d_{x^{2}-y^{2}}$ transitions, the satellite band originates from metal-to-ligand charge-transfer (MLCT) transitions. The calculated spectra were shifted by $20.0 \mathrm{eV}^{[6]}$
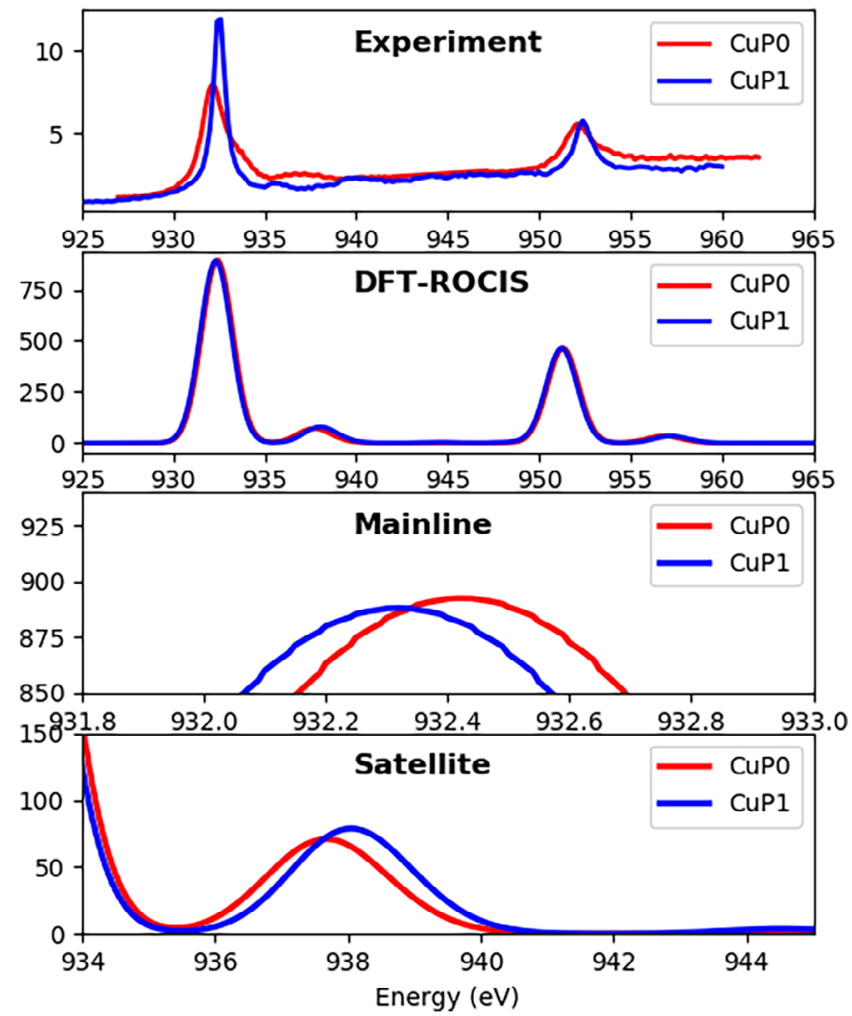

TAB LE 3 Calculated peak positions and intensities for the $\mathrm{Cu}$ L-edges of CuPO and CuP1

\begin{tabular}{llllll} 
& \multicolumn{2}{l}{ CuP0 } & & \multicolumn{2}{l}{ CuP1 } \\
\cline { 2 - 3 } Feature & Position & Intensity & & Position & Intensity \\
\hline $\mathrm{L}_{3}$ & 932.42 & 892.4 & & 932.32 & 888.0 \\
$\mathrm{~L}_{3}$ satellite & 937.67 & 70.8 & & 938.04 & 78.6 \\
\hline $\mathrm{L}_{2}$ & 951.34 & 462.4 & & 951.23 & 462.1 \\
\hline $\mathrm{L}_{2}$ satellite & 956.81 & 35.1 & 957.21 & 33.5 \\
\hline
\end{tabular}

Note: All energies are given in $\mathrm{eV}$.

and CuP1, respectively. Finally, it should be noted that a mismatch of the calculated and experimental satellite feature positions has been observed before and is to be expected with this methodology owing to its dependence on the amount of incorporated Hartree-Fock exchange in the used functional and the corresponding $c_{2}$ parameter. ${ }^{[6,68]}$

\subsection{3 | Restricted active space approach}

\section{Active space selection}

As the valence orbital space of the systems explored in the current study are composed of a large number of ligand-based $\pi$ orbitals, the active space selection and the treatment of core-excited state multiplets are far from being trivial. Calculating a wealth of complete multiplets (doubletdoublet- and doublet-quartet-type excitations) in the presence of dynamic electron correlation while maintaining a large valence active space that can capture all the essential information regarding the corresponding electronic structure unsurprisingly gives rise to an exhaustive computational cost. Therefore, the aforementioned RASCl approach, together with second-order n-electron valence perturbation theory to describe electron correlation (RASCI + NEVPT2), was used in combination with the recently introduced ASS1ST ${ }^{[69]}$ scheme to choose active spaces that include all strongly correlated orbitals but are as small as possible. ${ }^{[55]}$ In a nutshell, ASS1ST produces a set of quasirestricted natural orbitals for the internal and external orbital space. Based on the corresponding natural orbital occupation numbers (NOONs), the correlation strength of the natural 
orbitals is estimated, which in turn serves as the basis for the inclusion (or exclusion) of the natural orbitals in the active space. The starting point for the ASS1ST procedure in the current cases was an active space of nine electrons in five orbitals reflecting the $\mathrm{Cu}\left(3 \mathrm{~d}^{9}\right)$ configuration.

As depicted in Figure 9, two additional internal and external orbitals of CuPO are strongly correlated with occupation numbers of $\sim 1.92$, $\sim 1.94$, and $\sim 0.07$. As expected for extended conjugated $\pi$ systems, multiple further orbitals feature occupation numbers that considerably differ from both 0.0 and 2.0. However, owing to the aforementioned computational restrictions, only orbitals with a nonnegligible contribution from $\mathrm{Cu}$ orbitals were included, resulting in a $(7,7)$ active space. After a state-averaged optimization of all molecular orbitals with respect to all excited states up to $12 \mathrm{eV}$, as recommended previously, ${ }^{[25]}$ the active orbitals took the form displayed in Figure 10 . They comprise the singly occupied orbital with mostly $\mathrm{Cu} 3 \mathrm{~d}_{x^{2}-y^{2}}$ character and its bonding counterpart together with a pair of bonding and antibonding ligand $\pi$-orbitals and the $\mathrm{Cu}$ $4 \mathrm{~s}$ orbital. Analogous active orbitals were obtained for CuP1 (see Supporting Information).

\section{RASCI + NEVPT2}

Figure 11 shows the calculated Cu L-edge spectra for CuPO and CuP1 at the RASCI $(13,10)$ and RASCI $(13,10)+$ NEVPT2 levels of theory. Note that these active spaces incorporate the above discussed valence active orbitals and the $\mathrm{Cu} 2 \mathrm{p}$-orbitals in order to enable the calculation of the desired core excited states. Analogous to the DFT/ROCIS results (see above), the calculated spectra have to be shifted by -11.2 and 7.0 eV, respectively, to match the experimentally observed transition energies. As expected, the calculated spectra for CuPO and CuP1 exhibit great similarities. More precisely, both spectra feature single intense $L_{3}$ and $L_{2}$ bands with no visible shoulders and similar $L_{3} / L_{2}$ intensity ratios of 1.97 (CuP0) and 1.98 (CuP1). Hence, the RASCI $(13,10)+$ NEVPT2 results neither reproduce the experimentally observed shoulder on the high energy side of the $\mathrm{L}_{3}$-edge of CuPO nor its shift of $0.4 \mathrm{eV}$ relative to the $\mathrm{L}_{3}$-edge of CuP1.

The only considerable difference between the two calculated spectra is the position of the satellite feature on the high-energy side of the main $L_{3}$ edge. While it is predicted at $936.7 \mathrm{eV}$ for CuPO, it occurs at $935.6 \mathrm{eV}$ for CuP1 (see Table 4). In both cases, the position of the satellite features agrees remarkably well with the experimental spectra, with deviations in the order of $0.2 \mathrm{eV}$. We thus conclude that the chosen active space is well suited for the problem at hand. Nevertheless, it is important to note that a treatment of dynamic electron correlation on top of the $\mathrm{RASCl}$ calculation is imperative to obtain this level of accuracy as the same features are considerably shifted to higher energies ( $2 \mathrm{eV}$ ) at the RASCI level of theory (see Table 4). In agreement with our DFT/ROCIS results, our RASCI $(13,10)+N E V P T 2$ calculations indicate that the satellite features originate mostly from $2 \mathrm{p} \rightarrow \pi^{*}$ transitions for both CuPO and CuP1 (Figure 10). In a simple orbital energy-based picture, the considerable shift of the peak position toward a lower transition energy by $\sim 1 \mathrm{eV}$ for CuP1 might thus be associated with a lowering of the $\pi^{*}$ acceptor orbital

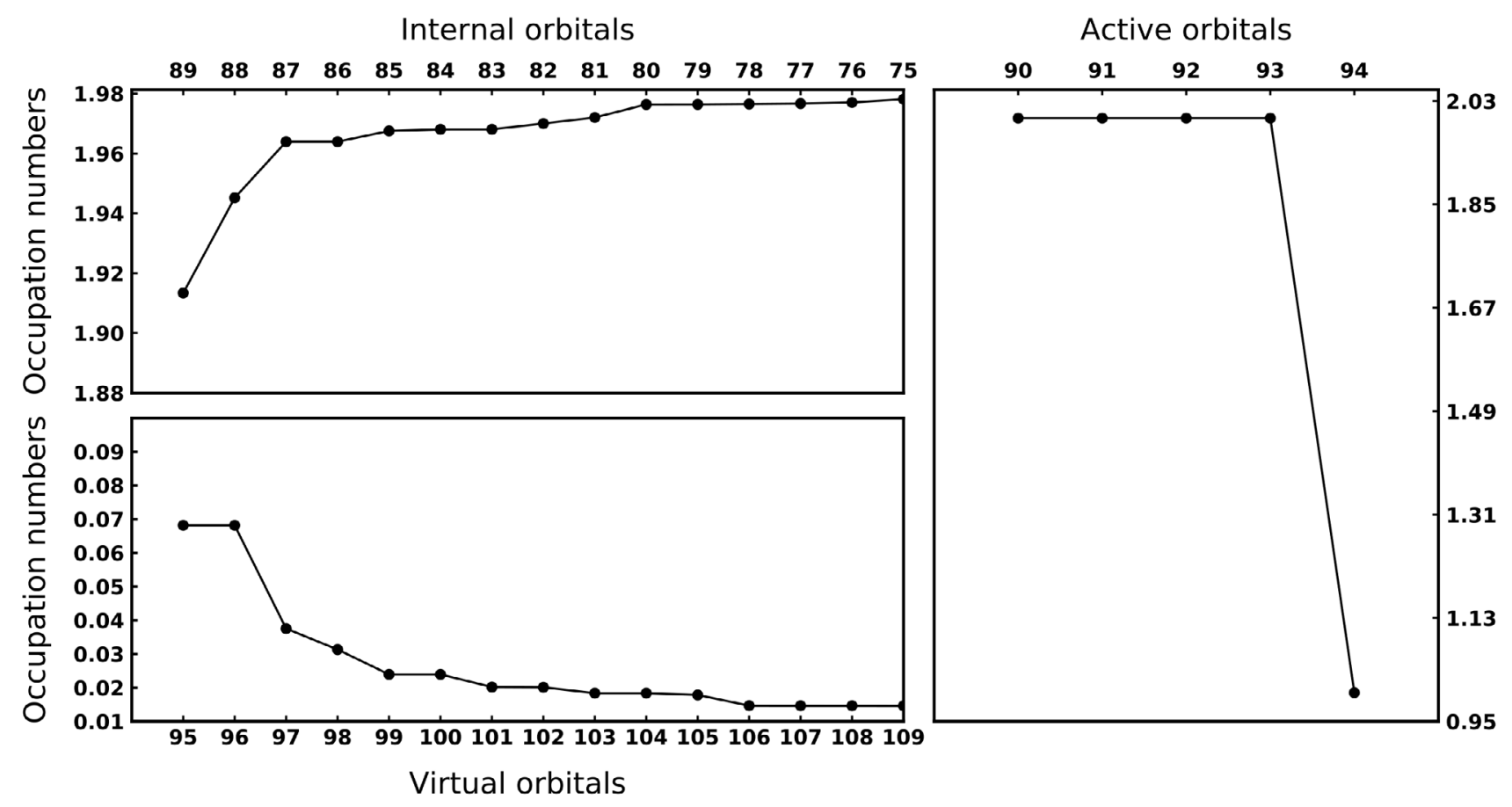

FIGURE 9 Natural orbital occupation number (NOON) distribution obtained from ASS1ST for CuPO. The corresponding NOON distribution for CuP1 can be found in the Supporting Information 
FIGURE 10 Optimized active orbitals for CuPO

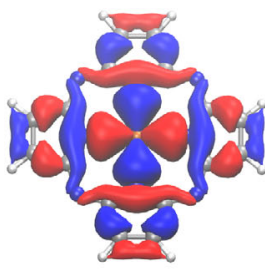

(A)

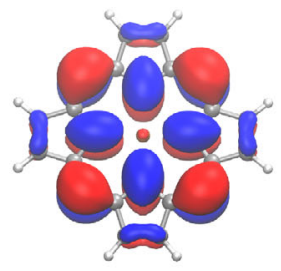

(B)

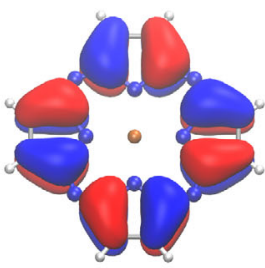

(C)

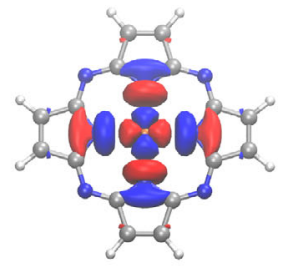

(D)

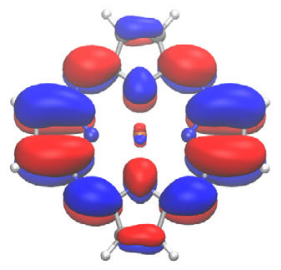

(E)

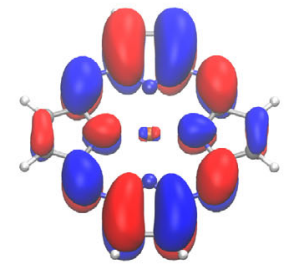

(F)

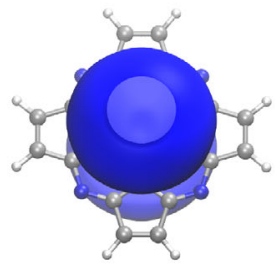

(G)
FIGURE 11 Experimental (top) and calculated (bottom) copper L-edge XAS for copper porphyrazine PO and phthalocyanine P1 using RASCI $(7,7)$ (top) and RASCI $(7,7)+$ NEVPT2 protocols

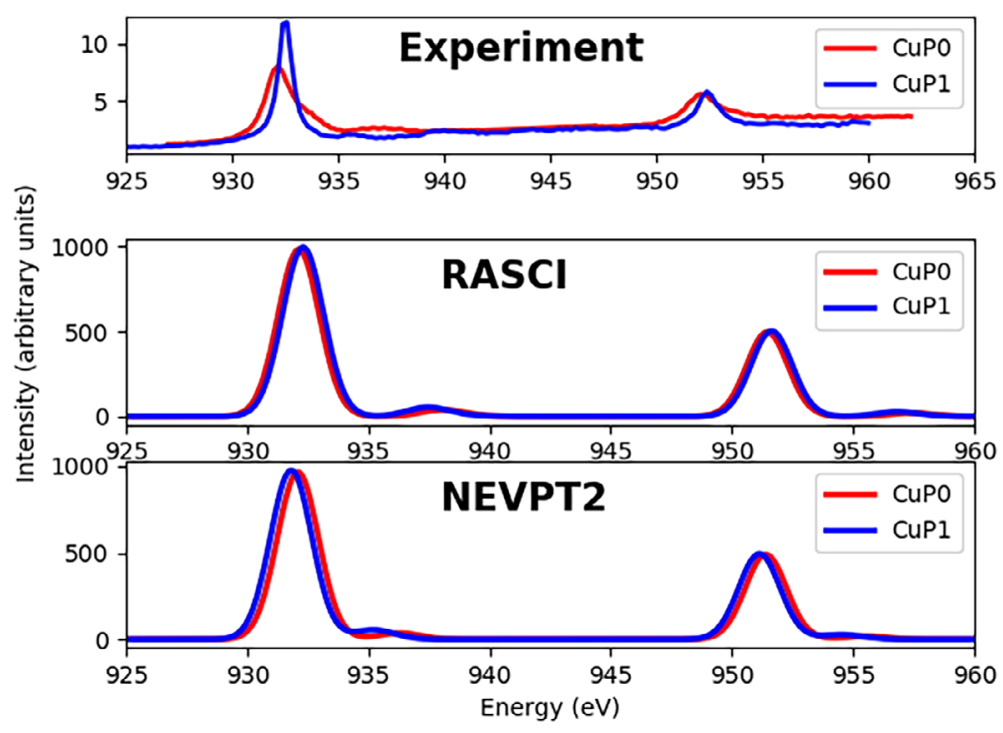

TAB LE 4 Calculated peak positions and intensities for the Cu L-edges of CuPO and CuP1 at the RASCI $(13,10)$ and RASCI $(13,10)+$ NEVPT2 levels of theory

\begin{tabular}{|c|c|c|c|c|}
\hline \multirow[b]{2}{*}{ Feature } & \multicolumn{2}{|l|}{ CuPO } & \multicolumn{2}{|l|}{ CuP1 } \\
\hline & Position & Intensity & Position & Intensity \\
\hline $\mathrm{RASCI}_{3}$ & 932.2 & 980.3 & 932.4 & 992.6 \\
\hline RASCI $L_{3}$ satellite & 938.2 & 39.4 & 937.5 & 54.2 \\
\hline NEVPT2 $L_{3}$ & 932.5 & 963.2 & 932.2 & 973.8 \\
\hline NEVPT2 $L_{3}$ satellite & 936.7 & 37.0 & 935.6 & 55.1 \\
\hline
\end{tabular}

Note: All energies are given in $\mathrm{eV}$.

energies on account of the enlargement of the conjugated $\pi$ system. Of course, if the active space does not include any ligand-centered $\pi$ (and $\pi^{*}$ ) orbitals but only metal-centered $3 \mathrm{~d}$ orbitals, none of the satellite features could possibly be predicted by the RASCI or RASCI + NEVPT2 calculations at all. Following this line of argument, the absence of the second satellite band for CuP1 in the calculated spectrum is probably due to the limited number of ligand-centered active orbitals. However, considering the steep increase in computational cost with the growing number of active orbitals and the potentially large number of required orbitals to reproduce this feature, we refrain from increasing the active space size beyond this point. 


\section{5 | SUMMARY AND CONCLUSIONS}

This work contains an analysis of the experimentally observed N K-edge and Cu L-edge of three Cu porphyrazine derivatives labeled CuP0, CuP1, and CuP2 based on electronic structure calculations. According to our (and previous) calculations, the electronic ground state of all three porphyrazines is dominated by a $\mathrm{Cu}\left(3 d^{9}\right)$ configuration with a singly occupied $3 d_{x^{2}-y^{2}}$ orbital, as is expected for square-planar Cull compounds.

For $\mathrm{N}$ K-edge spectra, remarkable agreement between calculated and experimental spectra was found in the investigated spectral region of up to 5 to $6 \mathrm{eV}$ above the rising edge. The first and most intense spectral feature A of that region originates from multiple transitions into lowlying $\pi^{*}$ and $\sigma^{*}$ transitions. Interestingly, the progressive broadening of the feature can be connected to a redistribution of contributions from the symmetrically inequivalent sets of $\mathrm{N}_{a z a}$ and $\mathrm{N}_{\text {pyr }}$ atoms. Higher-energy features $\mathrm{B}$ through $\mathrm{D}$ all correspond to $\mathrm{N} 1 \rightarrow \pi^{*}$ transitions. With increasing degree of benzolation, the larger number of available $\pi^{*}$ orbitals leads to a general broadening of these features. A general trend for features $\mathrm{B}$ to $D$ is that the higher the transition energy, the more the excited electron is located toward the periphery of the molecule.

Owing to the $\left(2 p^{5}\right)\left(3 d^{10}\right)$ final-state configuration of the corresponding excited states, the experimental Cu L-edge spectra of CuPO, CuP1, and CuP2 exhibit only little structure. Besides the main edge peaks, only a weakly resolved shoulder at the high-energy side of the $\mathrm{L}_{3}$ edge of CuPO and satellite features between the $L_{3}$ and $L_{2}$ edges are observed. From an experimental point of view, the origin of the shoulder is somewhat unclear. Interestingly, neither our DFT/ROCIS nor our RASCI + NEVPT2 calculations can reproduce the shoulder for CuPO. It may thus be inferred that it is due to effects that are outside of the current models, for example, axial ligation, interactions with the surface or other porphyrazines, etc. As all presented calculations refer to single molecules in the gas phase, such interactions and their influence on the core-electron spectra cannot be reproduced. Nevertheless, the applied model was sufficient to reproduce the structure observed at the N K-edge. The absence of any intense MLCT bands in the region of the $\mathrm{L}_{3}$-edge in the calculated spectra indicates that the shoulder rather originates from a splitting of the main $\mathrm{L}_{3}$ edge than from additional MLCT bands. Both theoretical approaches connect the satellite bands at 936.9 and $935.7 \mathrm{eV}$ for CuPO and CuP1, respectively, to transitions into low-lying $\pi^{*}$ orbitals. Accordingly, the observed energetical shift can be connected to an energetic lowering of the acceptor orbitals due to the extension of the conjugated $\pi$ system. From a technical point of view, it should be noted that, while the DFT/ROCIS method fails to give accurate transition energies for the satellite bands owing to its inherent dependence on the empirical $c_{2}$ parameter, the RASCI + SC-NEVPT2 results are in good agreement with the experimental values. Importantly, the treatment of dynamic electron correlation effects and the inclusion of ligandcentered orbitals in the active space are prerequisites for achieving this level of qualitative and quantitative accuracy.

\section{ACKNOWLEDGMENT}

Open access funding enabled and organized by Projekt DEAL.

\section{AUTHOR CONTRIBUTIONS}

Esma Boydas: Formal analysis; investigation; methodology; visualization; writing-review and editing. Bernd Winter: Investigation; writing-review and editing. David Batchelor: Conceptualization; investigation; resources; writing-review and editing. Michael Römelt: Conceptualization; formal analysis; funding acquisition; investigation; resources; writing-original draft.

ORCID

David Batchelor (D) https://orcid.org/0000-0001-9076-0780

Michael Roemelt (D) https://orcid.org/0000-0002-4780-5354

\section{REFERENCES}

[1] R. Golnak, S. I. Bokarev, R. Seidel, J. Xiao, G. Grell, K. Atak, I. Unger, S. Thürmer, S. G. Aziz, O. Kühn, B. Winter, E. F. Aziz, Sci. Rep. 2016,6 , 1.

[2] T. Glaser, B. Hedman, K. Hodgson, E. Solomon, Acc. Chem. Res. 2000, 33, 859.

[3] R. Sarangi, S. DeBeer George, D. J. Rudd, R. K. Szilagyi, X. Ribas, C. Rovira, M. Almeida, K. O. Hodgson, B. Hedman, E. I. Solomon, J. Am. Chem. Soc. 2007, 129, 2316.

[4] K. Lee, H. Wei, A. V. Blake, C. M. Donahue, J. M. Keith, S. R. Daly, Dalton Trans. 2016, 45, 9774.

[5] S. DeBeer, T. Petrenko, F. Neese, Inorg. Chim. Acta 2008, 361, 965.

[6] M. Roemelt, D. Maganas, S. DeBeer, F. Neese, J. Chem. Phys. 2013, 138, 204101.

[7] B. T. Thole, G. van der Laan, Phys. Rev. B 1988, 38, 3158.

[8] F. M. F. de Groot, J. C. Fuggle, B. T. Thole, G. A. Sawatzky, Phys. Rev. B 1990, 42, 5459.

[9] P. Bagus, H. Freund, H. Kuhlenbeck, E. Ilton, Chem. Phys. Lett. 2008, 455, 331.

[10] E. Stavitski, F. de Groot, Micron 2010, 41, 687.

[11] H. Ikeno, T. Mizoguchi, I. Tanaka, Phys. Rev. B 2011, 83, 155107.

[12] M. Haverkort, M. Zwierzycki, O. Andersen, Phys. Rev. B 2012, 85, 165113.

[13] D. Maganas, M. Roemelt, M. Hävecker, A. Trunschke, A. Knop-Gericke, R. Schlögl, F. Neese, Phys. Chem. Chem. Phys. $2013,15,7260$.

[14] D. Maganas, M. Roemelt, T. Weyhermüller, R. Blume, M. Hävecker, A. Knop-Gericke, S. DeBeer, R. Schlögl, F. Neese, Phys. Chem. Chem. Phys. $2014,16,264$.

[15] D. Maganas, S. DeBeer, F. Neese, Inorg. Chem. 2014, 53, 6374.

[16] K. Atak, R. Golnak, J. Xiao, M. Pflüger, T. Brandenburg, B. Winter, E. F. Aziz, Phys. Chem. Chem. Phys. 2015, $17,3409$. 
[17] S. S. N. Lalithambika, K. Atak, R. Seidel, A. Neubauer, T. Brandenburg, J. Xiao, B. Winter, E. F. Aziz, Sci. Rep. $2017,7,1$.

[18] S. Sreekantan Nair Lalithambika, R. Golnak, B. Winter, K. Atak, Inorg. Chem. 2019, 58, 4731.

[19] I. Josefsson, K. Kunnus, S. Schreck, A. Föhlisch, F. M. F. de Groot, P. Wernet, M. Odelius, J. Phys. Chem. Lett. 2012, 3, 3565.

[20] S. I. Bokarev, M. Dantz, E. Suljoti, O. Kühn, E. F. Aziz, Phys. Rev. Lett. 2013, 111, 083002.

[21] R. V. Pinjari, M. G. Delcey, M. Guo, M. Odelius, M. Lundberg, J. Chem. Phys. 2014, 141, 124116.

[22] M. Preuße, S. I. Bokarev, S. G. Aziz, O. Kühn, Struct. Dyn. 2016, 3, 062601.

[23] R. V. Pinjari, M. G. Delcey, M. Guo, M. Odelius, M. Lundberg, J. Comp. Chem. 2016, 37, 477.

[24] M. Guo, E. Källman, R. V. Pinjari, R. C. Couto, L. Kragh Sørensen, R. Lindh, K. Pierloot, M. Lundberg, J. Chem. Theory Comput. $2018,15,477$.

[25] A. Chantzis, J. K. Kowalska, D. Maganas, S. DeBeer, F. Neese, J. Chem. Theory Comput. 2018, 14, 3686.

[26] D. Maganas, J. K. Kowalska, M. Nooijen, S. DeBeer, F. Neese, J. Chem. Phys. 2019, 150, 104106.

[27] S. I. Bokarev, O. Kühn, WIREs Comp. Mol. Sci. 2020, 10, e1433.

[28] D. Pop, B. Winter, W. Freyer, R. Weber, W. Widdra, I. V. Hertel, J. Phys. Chem. B 2004, 108, 9158.

[29] G. De La Torre, P. Vazquez, F. Agullo-Lopez, T. Torres, Chem. Rev. 2004, 104, 3723.

[30] A. B. Sorokin, Chem. Rev. 2013, 113, 8152.

[31] L. Martín-Gomis, F. Fernández-Lázaro, Á. Sastre-Santos, J. Mater. Chem. A 2014, 2, 15672.

[32] P.-C. Lo, M. S. Rodríguez-Morgade, R. K. Pandey, D. K. Ng, T. Torres, F. Dumoulin, Chem. Soc. Rev. 2020, 49, 1041.

[33] D. Batchelor, D. Pop, B. Winters, W. Freyer, M. Sperling, B. Tepper, W. Widdra NEXAFS study of a family of Copper Porphyrazines. BESSY - Annual Report 20022002.

[34] D. Pop Ph.D. Thesis, Freie Universität Berlin 2003.

[35] J. Stöhr, D. Outka, Phys. Rev. B 1987, 36, 7891.

[36] D. Batchelor, U. Aygül, U. Dettinger, M. Ivanovic, A. Tournebize, S. Mangold, M. Forster, U. Scherf, H. Peisert, T. Chassé, Eur. Polym. J. 2016, 81, 686.

[37] D. R. Batchelor, R. Follath, D. Schmeißer, Nucl. Instrum. Methods Phys. Res., Sect. A 2001, 467, 470.

[38] O. Fuchs Ph.D. Thesis, Universität Würzburg, Fakultät für Physik und Astronomie 2009.

[39] F. Neese, WIREs Comp. Mol. Sci. 2018, 8, e1327.

[40] A. Becke, J. Chem. Phys. 1993, 98, 1372.

[41] A. D. Becke, J. Chem. Phys. 1993, 98, 5648.

[42] F. Weigend, R. Ahlrichs, Phys. Chem. Chem. Phys. 2005, 7, 3297.

[43] F. Neese, F. Wennmohs, A. Hansen, U. Becker, Chem. Phys. 2009, 356, 98.

[44] B. I. Dunlap, J. W. D. Connolly, J. R. Sabin, J. Chem. Phys. 1979, 71, 3396.

[45] O. Vahtras, J. Almlöf, M. W. Feyereisen, Chem. Phys. Lett. 1993, 213, 514.

[46] F. Neese, J. Comput. Chem. 2003, 24, 1740.

[47] F. Weigend, Phys. Chem. Chem. Phys. 2006, 8, 1057.

[48] E. Runge, E. Gross, Phys. Rev. Lett. 1984, 52, 997.

[49] J. Pipek, P. G. Mezey, J. Chem. Phys. 1989, 90, 4916.

[50] S. Hirata, M. Head-Gordon, Chem. Phys. Lett. 1999, 314, 291.

[51] E. v. Lenthe, E. J. Baerends, J. G. Snijders, J. Chem. Phys. 1993, 99, 4597.

[52] C. van Wüllen, J. Chem. Phys. 1998, 109, 392.

[53] S. DeBeer George, T. Petrenko, F. Neese, J. Phys. Chem. A 2008, 112, 12936.

[54] M. Roemelt, V. Krewald, D. A. Pantazis, J. Chem. Theory Comput. 2018, 14, 166.

[55] A. Khedkar, M. Roemelt, J. Chem. Theory Comput. 2019, 15, 3522.

[56] C. Angeli, R. Cimiraglia, S. Evangelisti, T. Leininger, J. Malrieu, J. Chem. Phys. 2001, 114, 10252.

[57] C. Angeli, R. Cimiraglia, J. Malrieu, J. Chem. Phys. 2002, 117, 9138.

[58] B. A. Hess, C. M. Marian, U. Wahlgren, O. Gropen, Chem. Phys. Lett. 1996, 251, 365.

[59] M. Douglas, N. M. Kroll, Ann. Phys. 1974, 82, 89.

[60] B. A. Hess, Phys. Rev. A 1986, 33, 3742.

[61] W. A. De Jong, R. J. Harrison, D. A. Dixon, J. Chem. Phys. 2001, 114, 48.

[62] N. B. Balabanov, K. A. Peterson, J. Chem. Phys. 2005, 123, 064107.

[63] N. B. Balabanov, K. A. Peterson, J. Chem. Phys. 2006, 125, 074110.

[64] G. L. Stoychev, A. A. Auer, F. Neese, J. Chem. Theory Comput. 2017, 13, 554.

[65] R. De Francesco, M. Stener, G. Fronzoni, J. Phys. Chem. A 2012, 116, 2885.

[66] A. J. Wallace, B. E. Williamson, D. L. Crittenden, Can. J. Chem. 2016, 94, 1163.

[67] M. Gouterman, J. Mol. Spectrosc. 1961, 6, 138.

[68] M. Roemelt, M. A. Beckwith, C. Duboc, M.-N. Collomb, F. Neese, S. DeBeer, Inorg. Chem. 2012, 51, 680.

[69] A. Khedkar, M. Roemelt, J. Chem. Theory. Comput. 2020, 16, 4993.

\section{SUPPORTING INFORMATION}

Additional supporting information may be found online in the Supporting Information section at the end of this article.

How to cite this article: Boydas EB, Winter B, Batchelor D, Roemelt M. Insight into the X-ray absorption spectra of Cu-porphyrazines from electronic structure theory. Int J Quantum Chem. 2020;e26515. https://doi.org/10.1002/qua.26515 